\title{
Evaluating Nahoon Beach and Canal Waters in Eastern Cape, South Africa: A Public Health Concern
}

\author{
Kingsley Ebomah ${ }^{1 *}$, Timothy Sibanda ${ }^{2}$, Martins Adefisoye ${ }^{1}$, Nolonwabo Nontongana ${ }^{1}$, \\ Uchechukwu Nwodo ${ }^{1}$, Anthony Okoh ${ }^{1}$
}

${ }^{1}$ SAMRC Microbial Water Quality Monitoring Centre, University of Fort Hare, Alice, South Africa ${ }^{2}$ Department of Environmental Science, UNISA Florida Campus, Roodepoort, South Africa

Received: 14 November 2017

Accepted: 12 February 2018

\begin{abstract}
Contamination of water resources by pathogens due to ever increasing anthropogenic activities and the related disease burden remains a major concern of water quality globally. This study evaluated the physicochemical and microbiological quality indices of Nahoon Beach and its canal waters in South Africa over a period of 12 months (September 2014 to August 2015). Water samples were collected bi-weekly from 6 sampling points ( 3 on the beach and 3 on the canal) and analyzed using standard methods. The physicochemical qualities of the beach and canal waters ranged as follows: turbidity 3.3-99.9 NTU, temperature $15-25^{\circ} \mathrm{C}, \mathrm{pH} 7-10$, electrical conductivity 30.0-741.7 $\mu \mathrm{S} / \mathrm{cm}$, and total dissolved solids $19-546 \mathrm{mg} / \mathrm{l}$. As for microbial counts of the water samples, Escherichia coli counts ranged in the order of $10^{1}-10^{3} \mathrm{CFU} / 100 \mathrm{ml}$ while Enterococcus counts varied in the order of $10^{1}-10^{2} \mathrm{CFU} / 100 \mathrm{ml}$. This study underscores the need for the protection of recreational water resources to safeguard public health.
\end{abstract}

Keywords: beach, microbiological, physicochemical, pollution, public health

\section{Introduction}

Limited and full contact recreational activities are popular in marine beaches in South Africa and elsewhere in the world. There are growing public health concerns that such contact recreational activities are leading to increasing incidents of the outbreak of swimming-related illness associated with exposure to microbial pathogens [1]. Marine recreational beaches get

*e-mail: kingsleyebomah.ke@gmail.com contaminated with microbial pathogens and parasites like viruses, bacteria, fungi, and protozoa through both point-source inputs such as sewage outfalls and nonpoint source inputs such as human bather shedding, sand resuspension, and stormwater runoff [2]. Most Western nations have enacted legislation that requires proactive monitoring of beach and water quality as a way of reducing the incidents of swimming-related illnesses [3], a move that has often resulted in public health advisories and beach closures owing to the detection of high levels of faecal indicator bacteria and/or dangerously high levels of heavy metals. 
The situation is usually different and worrisome in developing countries, where a combination of budgetary constraints and frail institutions result in the absence of surveillance schemes, allowing undetected pollution from stormwater runoff and domestic sewage and industrial effluent discharges to enter beaches. Of particular concern is the possibility of direct discharge of domestic sewage and industrial effluents into beaches, coupled with the absence of early warning systems to warn the public on the safety status of said beaches [3]. The greatest risks posed to swimmers are associated with accidental ingestion of water contaminated with enteric pathogens, the most common source of which is the discharge of wastewater contaminated with human or animal faeces [4]. Enterococci are one important member of the microbial gut communities of many animals whose abundance in human faeces coupled with their ease of growth in culture, greater salt tolerance than faecal coliforms, and correlation with human health outcomes in fresh and marine waters have led to their widespread use not only as tools for assessing recreational water quality but also as surrogates for pathogens and/or health effects in risk assessment and other modeling applications worldwide [5]. In addition to enterococci, $E$. coli is still widely used as a microbial water quality monitoring tool, especially where gastrointestinal pathogens are targeted [6-7].

Given the fundamental socio-economic role that recreational beaches play, it is important that the quality indices of beach waters are periodically determined with respect to their public health connotations. Epidemiological studies have established a relationship between surface water recreational activities such as swimming and boating and gastrointestinal illness, even though the severity of illness tends to vary across demographic characteristics, level of contamination, exposure intensity, and host susceptibility [8]. Because of the popularity of beaches with all population subgroups - including sensitive population groups such as the elderly, minor children, pregnant women, and the immunocompromised, who are more likely to succumb to infection if they come into contact with pathogen-contaminated water - there is need for beach water quality surveillance to include quantitative microbial risk assessment (QMRA) estimations. A study by Deflorio-Barker et al. [8] reported that acute gastrointestinal infections associated with recreating in marine water translated into higher hospital bills and higher numbers of working-age adults missing work than those reported for freshwater recreationists.

South Africa is endowed with recreational beaches on its Indian and Atlantic Ocean interfaces. On the Indian Ocean interface, one of the notable recreational beaches is Nahoon Beach, which is located in East London in the Eastern Cape Province of South Africa. This beach is an important resource for commercial activities such as fishing, recreational tourism, and water sport. A previous study by Igbinosa et al. [9] reported the presence of Vibrio species in treated final effluent being discharged by wastewater treatment facilities into the Indian Ocean between Nahoon and Eastern Beach, both in East London. Subsequent studies [10-12] also reported the isolation of Streptomyces spp. from the Nahoon Beach waters. In April 2013 there were reports of closure of Nahoon, which according to Schleyer [13]

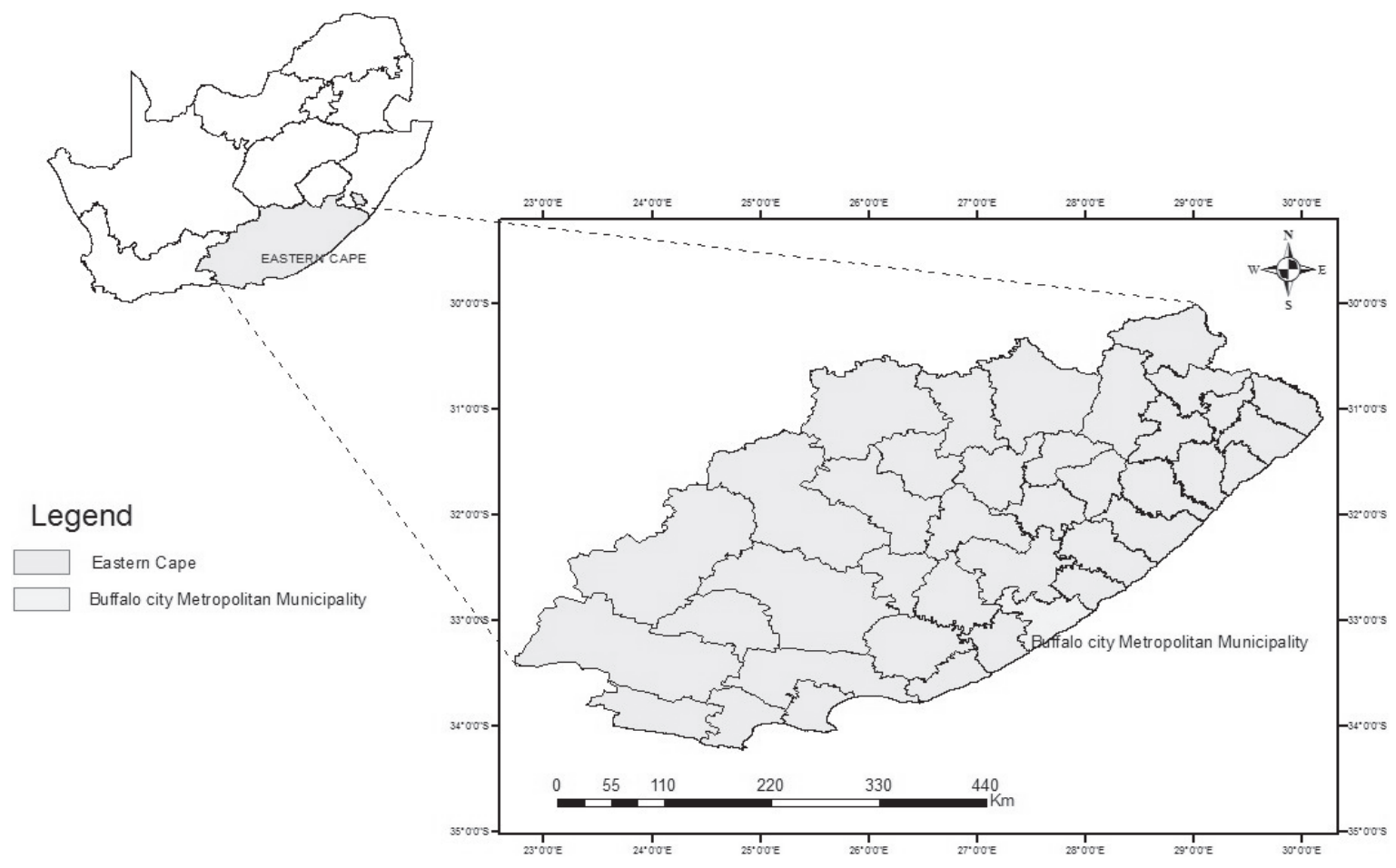

Fig. 1. Location of the study site. 
was due to beach water contamination from sewage outfalls. However, there is lack of consistency in the monitoring of beach water quality, leading to inadequate baseline information which may make it difficult to use predictive models in the future. In this paper, we report on the physicochemical and microbiological qualities of Nahoon Beach and its canal because, according to an article by Writer [14], although South Africa was awarded a rating of 58 blue flag status sites, Nahoon beach did not meet the standards (businesstech.co.za). Additionally, this paper reports on the estimated risk of infection to contact recreationists in Nahoon Beach and evaluates the perceived public health associated with recreational activity along the beach.

\section{Material and Methods}

\section{Description of Study Site}

Nahoon beach is situated in East London of Buffalo City Metropolitan Municipality in Eastern Cape, South Africa on the coast of the Indian Ocean $\left(32.99^{\circ} \mathrm{S}\right.$ and $27.95^{\circ} \mathrm{E}$ ). Nahoon canal is an extension of the Nahoon River, which flows into the beach. Nahoon beach boasts kilometres of mostly uncrowded and unpolluted sand and sea that produces some of the best waves, making it attractive to swimmers and surfers alike. Its reef provides fairly tough surfing conditions and has been preferred as a venue for many of the national surfing competitions. Complementing it is the Nahoon River mouth, a favourite location for fishermen and a safe swimming area for those who want to avoid pounding surf. Fig. 1 is a map showing Buffalo City Metropolitan Municipality in the Eastern Cape, South Africa.

However, final effluents from the East Bank Reclamation Works (sited in East London, South Africa), are released into the Indian Ocean at Bats Cave, which is in the middle of Nahoon and Eastern beach, with potential for these effluents to be spread by wave action onto both beaches.

\section{Sample Collection}

Triplicate water samples were collected fortnightly at knee depth using sterile $1 \mathrm{~L}$ bottles from 6 sampling points comprising 3 points each from the beach and the canal over a 12-month sampling period. The samples were immediately chilled by placing them in cooler boxes containing ice, transported to the Applied and Environmental Microbiology Research Group (AEMREG) Laboratory at the University of Fort Hare (Alice Campus), and analyzed within $6 \mathrm{~h}$ of collection.

\section{Physicochemical Analyses}

Five important physicochemical characteristics for beach water as outlined by USEPA [15] were determined on-site. The parameters, including $\mathrm{pH}$, temperature $\left({ }^{\circ} \mathrm{C}\right)$, electrical conductivity $(\mu \mathrm{S} / \mathrm{cm})$, and total dissolved solids (mg/l) were measured using a digital multiparameter system (Hanna; HI 9828), while turbidity was measured with the help of a digitally operated turbidimeter (HACH; 2100P).

\section{Microbiological Analyses}

Microbiological analysis of the beach water samples was done using the membrane filtration technique as previously described by Shash et al. [16]. Briefly, $100 \mathrm{ml}$ aliquots of the water samples were passed through $90 \mathrm{~mm}$ diameter, $0.45 \mu \mathrm{M}$ pore-sized filter membranes using a vacuum system. The filters were then aseptically transferred onto petri-dishes containing appropriated selective media for the isolation and enumeration of $E$. coli (Coliform Chromogenic agar) and Enterococcus sp. (Bile aesculin azide agar), respectively. Chromogenic agar plates were incubated at $37^{\circ} \mathrm{C}$ for $18-20 \mathrm{~h}$ while Bile aesculin azide agar were incubated at $37^{\circ} \mathrm{C}$ for $48 \mathrm{~h}$. Colonies that exhibited a characteristic blue colour in the Coliform Chromogenic agar medium were enumerated as presumptive E. coli, while those that exhibited a brown colour in the Bile aesculin azide agar medium were enumerated as presumptive Enterococcus sp. Results were reported as colony forming units per $100 \mathrm{ml}(\mathrm{CFU} / 100 \mathrm{ml})$ of beach water sample analysed. Over the course of 12 months, a sum total of 522 presumptive isolates were collected and analysed. The presumptive isolates were stored in $25 \%$ glycerol at $-80^{\circ} \mathrm{C}$ for further analysis.

\section{Molecular Identification}

Bacterial DNA was extracted from presumptive E. coli and Enterococcus isolates using the boiling method [17]. The target gene uidA (Forward primer: 5'AAAACGGCAAGAAAAAGCAG-3'; Reverse primer: 5'-ACGCGTGGTTAACAGTCTTGCG-3') was used for the identification of $E$. coli with an amplicon size of $147 \mathrm{bp}$. The PCR conditions for the molecular identification of the presumptive $E$. coli isolates were as follows: 5 minutes initial denaturation at $94^{\circ} \mathrm{C}$ followed by 35 cycles of $95^{\circ} \mathrm{C}$ for 30 seconds, $58^{\circ} \mathrm{C}$ for 1 minute, $72^{\circ} \mathrm{C}$ for 1 minute, and final extension at $72^{\circ} \mathrm{C}$ for 8 minutes. The target gene $\operatorname{SodA}$ (Forward primer: 5'TACTGACAAACCATTCATGATG3'; Reverse primer: 5'AACTTCGTCACCAACGCGAAC-3') was used for the identification of Enterococcus spp. with an amplicon size of $112 \mathrm{bp}$ [18]. The PCR conditions for the molecular identification of the presumptive Enterococcus isolates were as follows: 5 minutes initial denaturation at $94^{\circ} \mathrm{C}$ followed by 35 cycles of $95^{\circ} \mathrm{C}$ for 30 seconds, $58^{\circ} \mathrm{C}$ for 1 minute, $72^{\circ} \mathrm{C}$ for 1 minute, and final extension at $72^{\circ} \mathrm{C}$ for 8 minutes. The speciation of Enterococcus was carried out using PCR technique with primer sequences and PCR conditions as follows: E. faecalis (Forward primer: 
5'-TCAATGCAGTTCCGTTATCAGTT3'; Reverse primer: 5'GTAAAGTCCGTTACCCCAACCTG-3'), 30 seconds initial denaturation at $95^{\circ} \mathrm{C}$ followed by 28 cycles of $51^{\circ} \mathrm{C}$ for 30 second, $68^{\circ} \mathrm{C}$ for 9 minutes, $68^{\circ} \mathrm{C}$ for 2 minutes, final extension at $72^{\circ} \mathrm{C}$ for 1 minute, and E. faecium (Forward primer: 5'-GGCGACAGATTATACCGTGC-3'; Reverse primer: 5'-CGGTCTCTATATTCCCTGTT-3'); 2 minutes initial denaturation at $94^{\circ} \mathrm{C}$ followed by 35 cycles of $94^{\circ} \mathrm{C}$ for 1 minute, $55^{\circ} \mathrm{C}$ for 1 minute, $72^{\circ} \mathrm{C}$ for 1 minute, and final extension at $72^{\circ} \mathrm{C}$ for 5 minutes with amplicon sizes of $360 \mathrm{bp}$ and $215 \mathrm{bp}$, respectively. The PCR products $(5 \mu \mathrm{L})$ were stained with $0.5 \mathrm{mg} / \mathrm{L}$ ethidium bromide and then resolved on $1.5 \%$ agarose gel with the use of electrophoresis in $0.5 \times$ TBE (Tris-borateEDTA) buffer. The image was then visualized under a BioDoc-It ultraviolet transilluminatore (UVP Upland, CA, USA).

\section{Risk of Infection Analysis}

Quantitative Microbial Risk Assessment (QMRA) was used to estimate the probability of infection and assess the health risk it posed to the public by the presence of both E. coli and Enterococcus in Nahoon Beach and Nahoon Canal waters. For the purposes of risk of infection estimation, data was clustered into wet and dry categories based on hydrological events. Data for April to September was grouped into the "dry" category while data for October to March was grouped into the "wet" category.

\section{Exposure Assessment}

In this study, exposure analysis was based on two principles: (1) the average concentration of each of E. coli and Enterococcus group bacteria in the water samples from each of the six sampling points on the beach and canal, and (2) the average incidental volume of water consumed per individual during recreational activities. Since there was no questionnaire conducted to ascertain the exact volumes of water consumed during recreation, a ten-fold incremental scale ranging from $0.1 \mathrm{ml}$ to $100 \mathrm{ml}$ was used, since $100 \mathrm{ml}$ has been reported by Steyn et al. [19] to represent involuntary volume of water ingested during full-contact recreational activity.

\section{Dose-response}

For enterococci, the probability of gastrointestinal (GI) illness from incidental ingestion of enterococcicontaminated water was estimated with an exponential exposure-response model of Haas et al. [20], reported by Stone et al. [21] as follows:

$$
\begin{gathered}
p(\mathrm{illl})_{\text {day }}=\left\{1-\exp \left(-D_{\text {oral }} / \mathrm{k}\right)\right\} \cdot \Psi \\
D_{\text {oral }}=I_{\text {oral }} \cdot(c / 100)
\end{gathered}
$$

$\ldots$ where $p(\text { ill })_{\text {day }}$ is the daily probability of GI illness, $D_{\text {oral }}$ is the number of enterococci as CFU ingested per day, $k$ is an Enterococcus-specific infectivity parameter (1442), $\Psi$ is the ratio of illness to infection (0.2), $I_{\text {oral }}$ is a random variable for water ingestion rates $(\mathrm{ml} / \mathrm{d})$, and $c$ is a random variable drawn from the distribution of enterococci density (CFU/100 ml).

For E. coli, a beta-Poisson dose-response model was used to calculate the probability of infection $(P i)$ resulting from the incidental ingestion of $0.1 \mathrm{ml}, 1 \mathrm{ml}$, $10 \mathrm{ml}$, and $100 \mathrm{ml}$ of beach water as described by the World Health Organization [22]. The $1 \mathrm{ml}$ value for incidental ingestion was defined as the volume (droplets resulting from splashing) of water accidentally swallowed through the mouth or inhaled through the nose and subsequently swallowed [23]. Since E. coli isolated from environmental samples could include non-pathogenic strains, the $P i$ was calculated based on the assumption that $8 \%$ of average E. coli counts are pathogenic [24].

The equation used for calculating the $P i$ due to ingestion of $0.1 \mathrm{ml}, 1 \mathrm{ml}, 10 \mathrm{ml}$, and $100 \mathrm{ml}$ of beach water based on a single exposure was given as follows:

$$
P_{i}=1-(1+\mathrm{d} / \boldsymbol{\beta})^{-\alpha}
$$

...where $d=$ daily exposure and $\beta$ (2.473) and $\alpha(0.395)$ are organism-specific equation parameters [25].

\section{Statistical Analysis}

All statistics were performed using Microsoft Excel 2010 and SPSS Version 20 (Statistical Package for the Social Sciences - IBM Corporation, Armonk, New York, USA). The arithmetic mean of the E. coli counts was considered to appropriately describe the summary of the microbial concentration and was thus used for calculating the microbial risk as described elsewhere $[20,26]$. A single-factor analysis of variance (ANOVA) was used to compare the mean E. coli counts and Enterococcus spp. between September 2014 and August 2015, and also to compare their probabilities of infection. All statistics were considered significant at an alpha $(\alpha)$ of 0.05 .

\section{Results}

\section{Physicochemical Characteristics of Nahoon} Beach and Canal Waters

The mean values of physicochemical parameters for the three sampling points on Nahoon Beach are presented in Table 1, while Table 2 shows the mean values for the three sampling sites on the canal. The range of physicochemical parameter readings and the concomitant national and international guideline readings are presented in Table 3. The values of the physicochemical parameters measured in both 


\begin{tabular}{|c|c|c|c|c|c|c|}
\hline & & 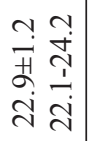 & 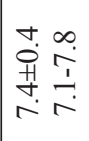 & 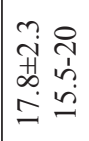 & 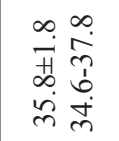 & 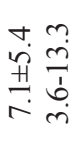 \\
\hline & & 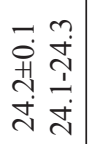 & $\begin{array}{ll}m & n \\
0 & n \\
+1 & i \\
r & i\end{array}$ & 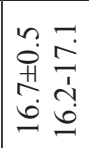 & 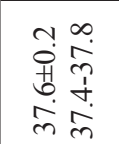 & 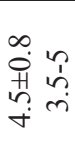 \\
\hline & & 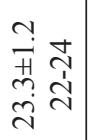 & 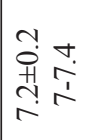 & $\frac{2}{3}$ & 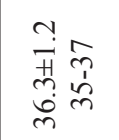 & 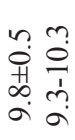 \\
\hline & & 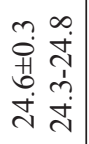 & 家 $\frac{2}{i}$ & 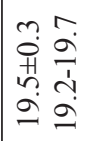 & 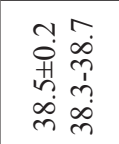 & 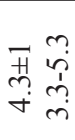 \\
\hline & & 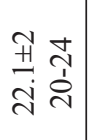 & 윰 & 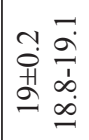 & 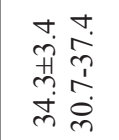 & 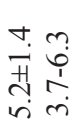 \\
\hline & & 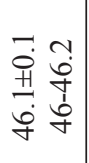 & 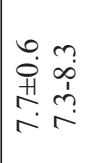 & 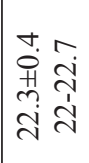 & 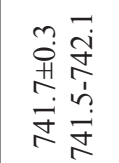 & 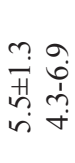 \\
\hline & & 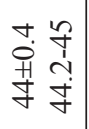 & 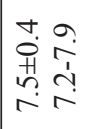 & 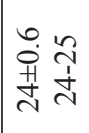 & 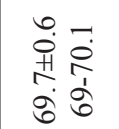 & 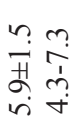 \\
\hline & 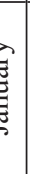 & 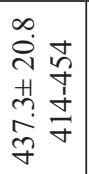 & $\mid \begin{array}{ll}\infty & n \\
\dot{1} & n \\
+1 & 0 \\
\infty & \infty\end{array}$ & 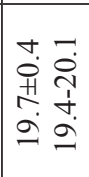 & 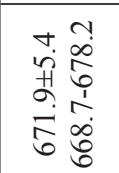 & 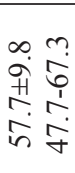 \\
\hline & & 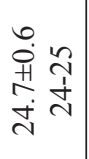 & 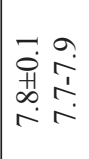 & 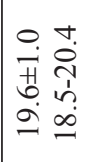 & 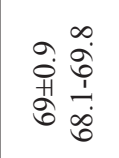 & 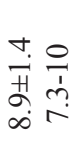 \\
\hline & & 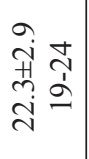 & 吾 & 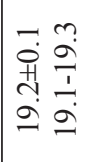 & 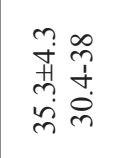 & 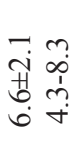 \\
\hline & & 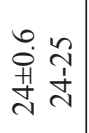 & 요를 & 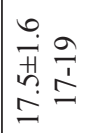 & 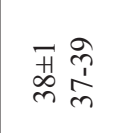 & 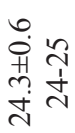 \\
\hline & 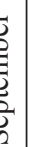 & 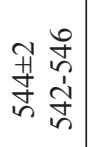 & 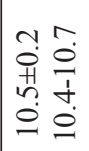 & 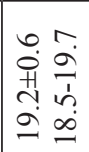 & 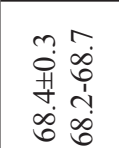 & 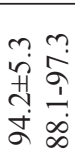 \\
\hline & & 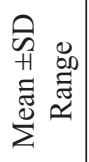 & 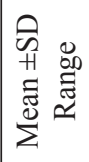 & 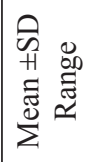 & 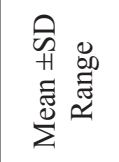 & 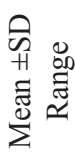 \\
\hline & & 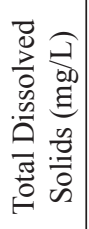 & 壳 & 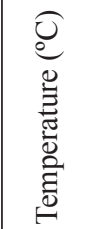 & 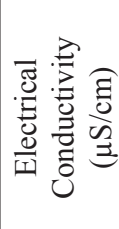 & 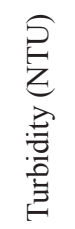 \\
\hline
\end{tabular}

\begin{tabular}{|c|c|c|c|c|c|}
\hline $\begin{array}{l}\vec{v} \\
\overrightarrow{5} \\
\vec{z}\end{array}$ & 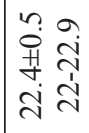 & 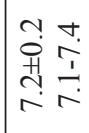 & $\begin{array}{ll}n & n \\
0 & 0 \\
0 & 0 \\
n & 1 \\
0 & 0 \\
0 & 0\end{array}$ & 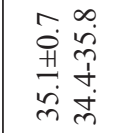 & 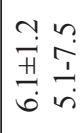 \\
\hline$\frac{\lambda}{3}$ & 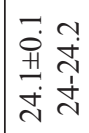 & 苦宰 & 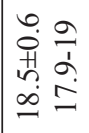 & 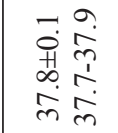 & 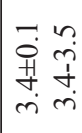 \\
\hline$\Xi$ & 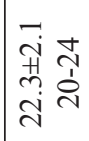 & 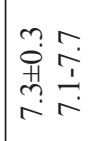 & 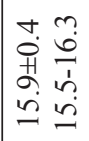 & 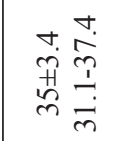 & 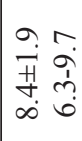 \\
\hline$\sum_{\bar{L}}^{\mathrm{I}}$ & 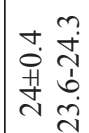 & \begin{tabular}{lr}
$m$ & $r$ \\
0 & \multicolumn{1}{r}{} \\
$m$ & $\frac{1}{n}$
\end{tabular} & 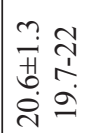 & 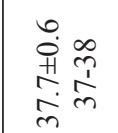 & 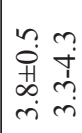 \\
\hline 总 & 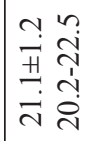 & 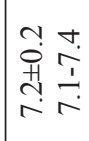 & 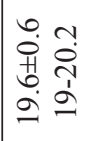 & 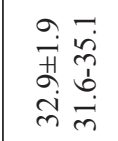 & 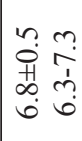 \\
\hline 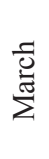 & 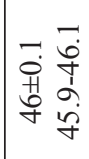 & 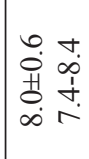 & 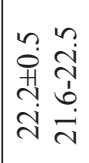 & 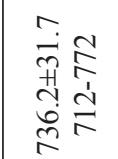 & 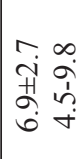 \\
\hline 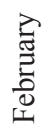 & 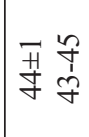 & 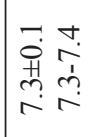 & 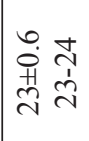 & 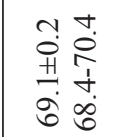 & 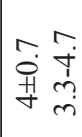 \\
\hline $\begin{array}{l}\vec{\Xi} \\
\stackrel{\Xi}{\Xi} \\
\text { 吾 }\end{array}$ & 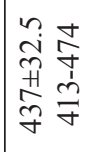 & 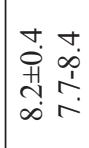 & 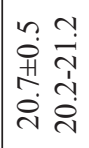 & 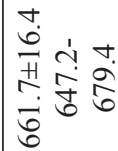 & 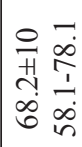 \\
\hline $\begin{array}{l}\overline{\bar{\delta}} \\
\text { है } \\
\bar{\Xi} \\
\text { ڤ̆ }\end{array}$ & 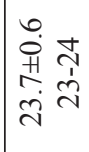 & 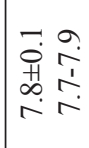 & 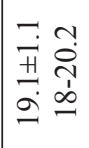 & 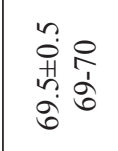 & 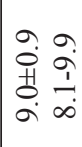 \\
\hline $\begin{array}{l}\text { ठठ } \\
\text { है } \\
\text { हे } \\
\text { z }\end{array}$ & 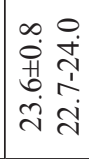 & 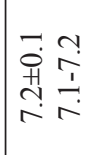 & 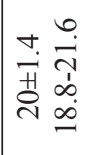 & 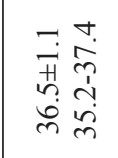 & 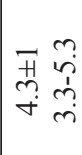 \\
\hline $\begin{array}{l}\dot{0} \\
\stackrel{0}{0} \\
\overline{0} \\
0\end{array}$ & 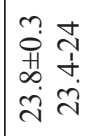 & $\vec{c}$ & 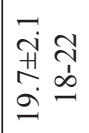 & 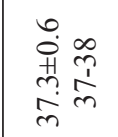 & 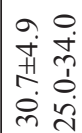 \\
\hline 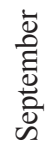 & 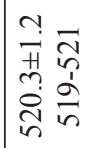 & 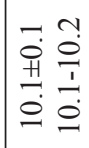 & 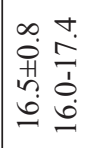 & 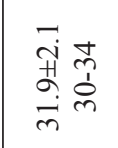 & 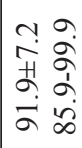 \\
\hline & 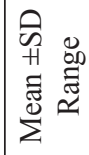 & 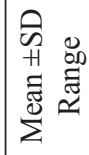 & 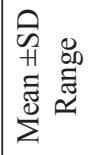 & 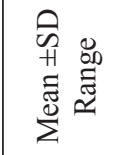 & 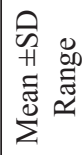 \\
\hline 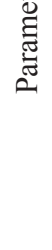 & 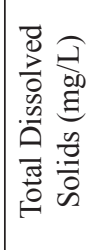 & 兑 & 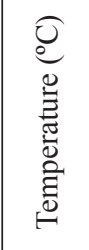 & 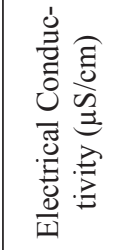 & 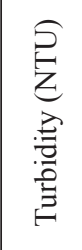 \\
\hline
\end{tabular}




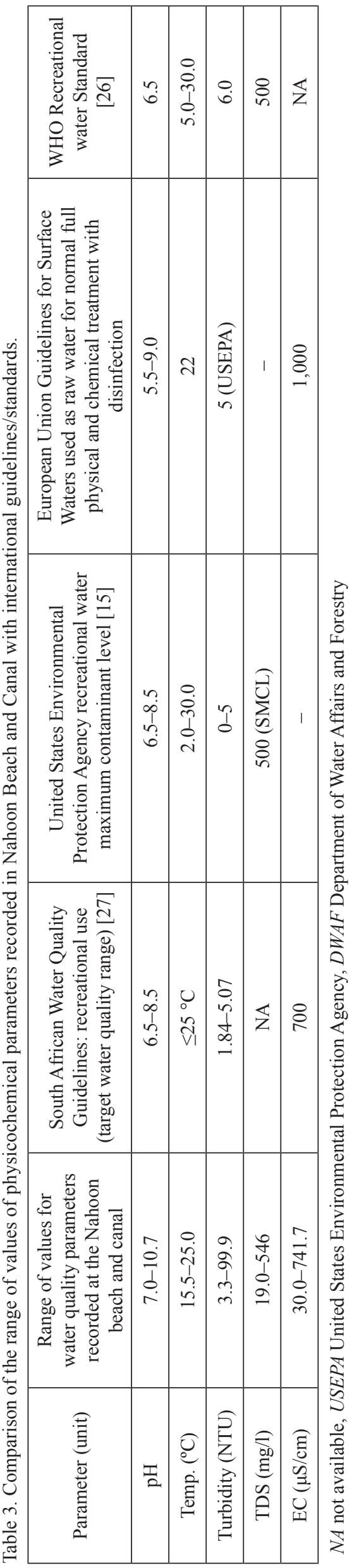

the beach and canal were mostly consistent with levels established by the health authorities, with the exception of turbidity. Water turbidity for all evaluated samples ranged from 3.3 to $97.3 \mathrm{NTU}$ for the beach and 3.3 to 99.9 NTU for the canal water. pH levels were within acceptable guidelines for most the sampling period, with averages ranging from 7 to 10.7 and from 7 to 10.2 - except for the month of September 2014, which had mean values of 10.5 and 10.1 for the beach and canal waters, respectively.

At all sampling sites (both beach and canal), turbidity ranged between 3.3 and 99.9 NTU, with the highest turbidity of 99.9 NTU and 97.3 NTU for the canal and beach water, respectively, occurring in the month of September. The lowest observed turbidity was $3.3 \mathrm{NTU}$, which was in the months of February, May, and November. The concentration of total dissolved solids (TDS) ranged between 19 and $546 \mathrm{mg} / \mathrm{L}$ for the beach and 20 and $521 \mathrm{mg} / \mathrm{L}$ for the canal water.

\section{Microbiological Characteristics of the Nahoon Beach and Canal Waters}

A total of 260 presumptive E. coli isolates and 172 presumptive Enterococcus strains were isolated from both the beach and canal waters. Of the presumptive E. coli isolates, $41.2 \%$ were confirmed positive for the uidA gene, while $59 \%$ of the presumptive Enterococcus strains were confirmed positive for the SodA gene, which positively identifies Enterococcus spp. Of the 102 enterococci isolates, 22 strains were identified to species level, being 14 strains for E. faecalis and 8 strains for E. faecium. The mean month-by-month bacterial counts obtained for both the beach and canal samples are presented in Fig. 2. The highest E. coli counts $(235 \mathrm{CFU} / 100 \mathrm{ml})$ were observed in January 2015, while the lowest counts $(4 \mathrm{CFU} / 100 \mathrm{ml})$ were in June and July 2015. The highest enterococci counts $(50 \mathrm{CFU} / 100 \mathrm{ml})$ were obtained in September 2014 while the lowest counts $(7 \mathrm{CFU} / 100 \mathrm{ml})$ were in June 2015. Further molecular analyses were held and some of the E. coli enterococci strains were found to be pathogenic but were not highlighted in this paper.

\section{Probability of Infection (E. coli) and Probability of Illness (enterococci) Estimations}

Fig. 3a) and b) summarizes the probability of infection $(P i)$ with pathogenic $E$. coli and enterococci respectively due to incidental ingestion of $0.1 \mathrm{ml}, 1 \mathrm{ml}$, $10 \mathrm{ml}$, or $100 \mathrm{ml}$ of recreational beach water from Nahoon Beach and canal waters during water sports such as swimming, surfing, or boating. Based on the accidental ingestion of different volumes of beach water, it was observed that E. coli posed a greater risk of infection to swimmers than enterococci. Furthermore, the risk of infection posed by E. coli was heightened during the wet season as compared to 


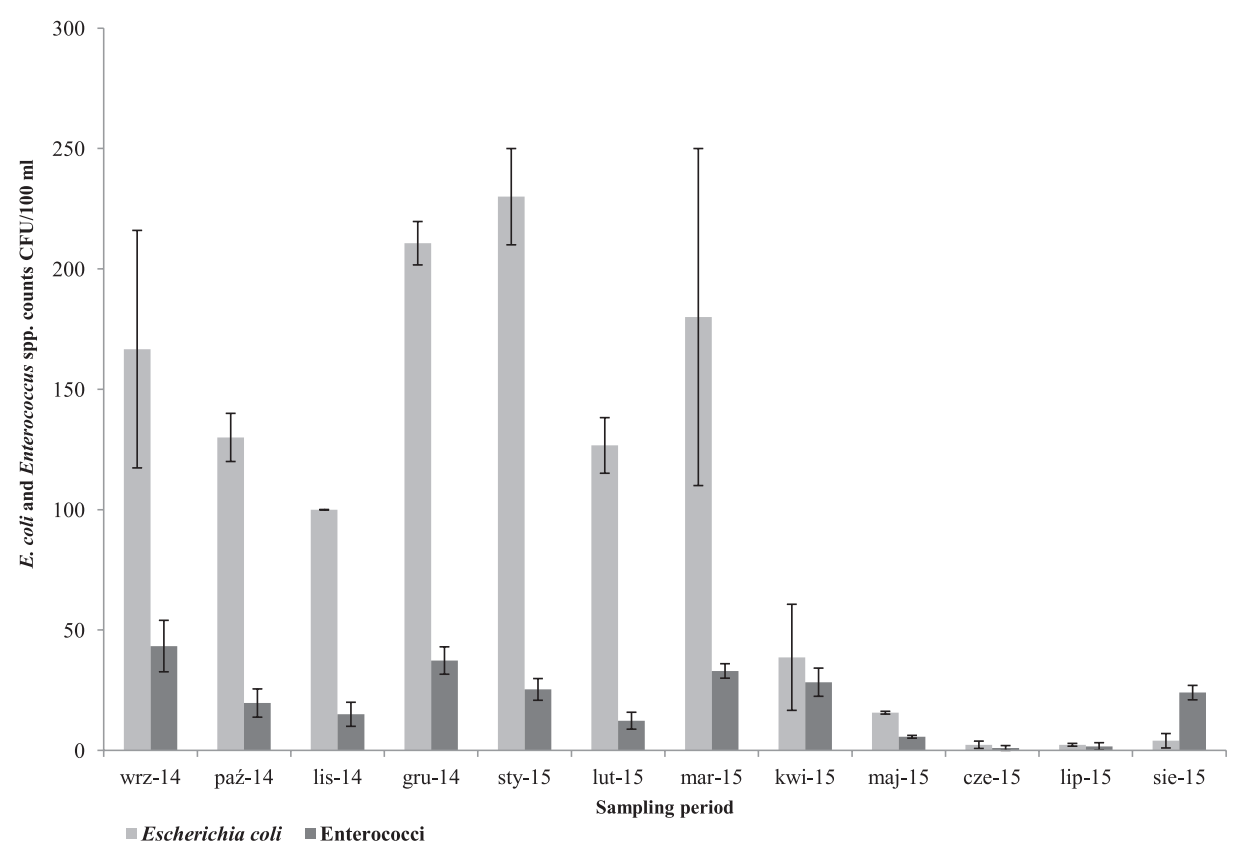

Fig. 2. E. coli and enterococci counts (CFU/100 ml) in beach and canal water samples, September 2014 to August 2015.

a)

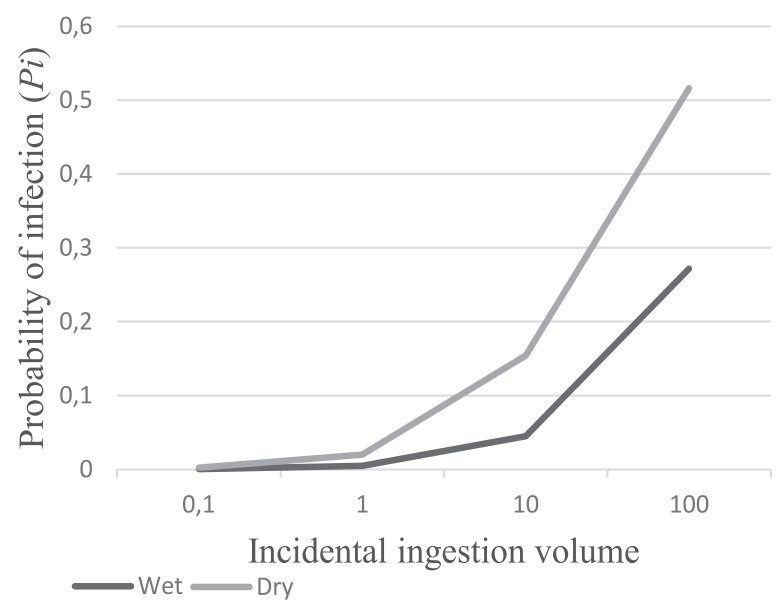

b)

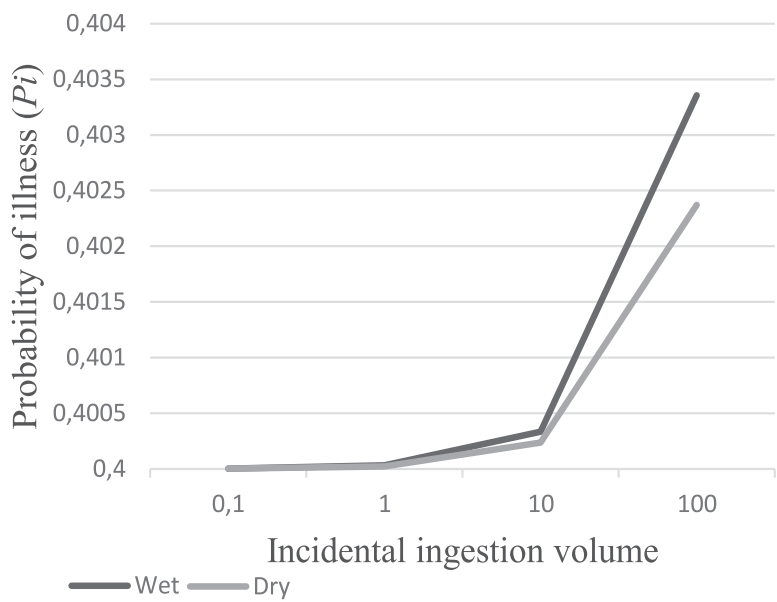

Fig. 3. a) Probability of infection with E. coli and b) probability of illness from enterococci exposure after incidental ingestion of $0.1 \mathrm{ml}, 1 \mathrm{ml}, 10 \mathrm{ml}$, and $100 \mathrm{ml}$ of recreational beach water. the dry season. Significant differences of the risk were observed with an increase in the volume of water consumed, starting at $10 \mathrm{ml}$ up to $100 \mathrm{ml}$, where the risk of infection for the wet category $(0.52)$ became nearly twice as much as the risk for the dry category $(0.28)$.

The probability of illness estimations from Enterococcus exposure assumed a similar trend to the risk of infection by E. coli, with heightened risk being observed during the wet season compared to the dry season. While the risk posed by enterococci was significantly less than that posed by $E$. coli at lower volumes $(0.1 \mathrm{ml}, 1 \mathrm{ml}$, and $10 \mathrm{ml})$, the risk of infection from Enterococcus exposure during the wet season astronomically increased at the consumption of $100 \mathrm{ml}$ of sea water, and became significantly higher than the risk posed by E. coli at $100 \mathrm{ml}$ during the dry season. However, contrary to the trend observed for E. coli, there were no significant differences in the risk of infection posed by Enterococcus in the wet season and in the dry season.

\section{Discussion}

The pollution level and anthropogenic pressure on receiving water systems can be measured by the physicochemical quality evaluation of the concerned systems. In this study, five physicochemical characteristics of the Nahoon Beach water samples, including $\mathrm{pH}$, temperature, turbidity (NTU), total dissolved solids (TDS), and electrical conductivity (EC) were assessed over a 12-month sampling period. Changes in water $\mathrm{pH}$ can be indicative of either an industrial pollutant or photosynthetic activity, usually as a result of eutrophication. The monitoring of $\mathrm{pH}$ is 
recommended as an assessment of the health of aquatic ecosystems, safety of recreational waters, and quality of industrial discharges, intakes, and storm water overflows [27].

The $\mathrm{pH}$ of uncontaminated water generally shows values ranging from 6.5 to 8.5 [28-29]. In this study, however, the $\mathrm{pH}$ values were at an average of 8.7 in the month of January for Nahoon Beach and an average of 10.0 in September for both the beach and canal samples. For recreational water, USEPA [15] recommends a $\mathrm{pH}$ range of 6.5-8.5, and contact with water whose $\mathrm{pH}$ is higher than this has been linked to primary irritation of the skin (although the mechanism is unclear) [30]. While it may not have been possible to pinpoint the actual cause of the $\mathrm{pH}$ surge in September, this increase coincided with stormy weather, which resulted in significant stormwater runoff to the beach. It could be that the stormwater brought with it some inorganic substances which possibly altered the $\mathrm{pH}$ balance. In line with this argument, Farrant and Hewson [31] have previously reported that high $\mathrm{pH}$ values in receiving water bodies are due to stormwater runoff with contaminants from both vehicular and construction sources.

High turbidity was generally recorded in the months of September and October (which fall into the spring season according to South African seasons). Generally, an increase in turbidity can be a result of an increase in substances that form colloids as compared to dissolved solids; nevertheless, an increase in total dissolved solids can also be linked to high turbidity in the water body [32]. Increased turbidity in water is frequently associated with the prospect of microbiological contamination [33], as high turbidity tends to shield microorganisms from natural sunlight inactivation. Our findings seem to support this view, as the highest counts for both $E$. coli and Enterococcus were recorded in September - the month in which the highest turbidity was also recorded. This finding suggests that microbial risk is heightened in the period after stormy weather, when significant inflows are received into the beach. Similar findings and inferences were made by Brady et al. [34], who even suggested that recreational microbial water quality can be predicted using turbidity.

Electrical conductivity (EC) values were compliant with the DWAF [29] guidelines for recreational waters (except in the month of March 2015). Some states, regions, and agencies have regularly set a TDS maximum as an option to conductivity in water quality monitoring, with conductivity largely a derivative of TDS content [35, 36-37]. Concomitantly, a rapid rise in conductivity in any water body can indicate pollution. According to Kemker [38], stormwater runoff, agricultural runoff, or perhaps a sewage outflow will cause EC to rise due to the surplus chloride, phosphate, nitrate ions, and other constituents such as alkaline metals (sodium). EC and salinity both can vary greatly amid various bodies of water. Several freshwater streams as well as lakes and rivers have low salinity and conductivity figures. The oceans, on the other hand, have high conductivity and salinity owing to their high quantity of dissolved salts. Poma et al. [39] showed strong positive correlations between water conductivity and viruses and parasites, chiefly norovirus, adenovirus, and Microsporidium $\mathrm{sp}$. We propose that if conductivity has to be used as a model for viruses and parasites, a baseline conductivity value has to be established by means of sustained assessments over time. Future water quality assessments will then have to take into consideration the established baseline value, and in all instances where this value is exceeded, assessments for viral and parasitological contamination will have to be done.

Generally, the temperature values observed in this current study ranged as follows: Nahoon Beach $\left(15^{\circ} \mathrm{C}-25^{\circ} \mathrm{C}\right)$ and Nahoon Canal $\left(15^{\circ} \mathrm{C}-24^{\circ} \mathrm{C}\right)$ fell within the required temperature recommendations of $2^{\circ} \mathrm{C}-30^{\circ} \mathrm{C}$ for recreational waters, where complete contact usage is permitted [15]. Considerably lower temperature $\left(15-19^{\circ} \mathrm{C}\right)$ was constantly recorded during winter season at all sampling points compared to summer water temperatures $\left(18-25^{\circ} \mathrm{C}\right)$.

Microbiological analysis showed that the E. coli counts exceeded $130 \mathrm{CFU} / 100 \mathrm{ml}$, which is the maximum accepted guideline for recreational water $[15$, 28-29]. Similarly, Enterococcus sp. counts were observed to exceed the recommended limit of $35 \mathrm{CFU} / 100 \mathrm{ml}$ for marine water [15, 28-29]. However, there was a reduction in the counts during winter, possibly due to a reduction in the number of swimmers and beach goers, which directly reduces real-time faecal bacteria shedding into the beach water and/or sand. During the wet (summer) season, beach water quality was poor judging from analysis made on both physicochemical and bacteriological parameters. This could have been a result of both meteorological and anthropogenic input, since temperatures during this period tend to favor beach activity, resulting in increased numbers of people visiting the beach. Elevated E. coli and Enterococcus concentrations in water can be indicative of the possible presence of potential pathogens belonging to these genera and others, including viral pathogens. McNinch et al. [40] and Göransson et al. [41] observed that during rainfall, the microbial loads of running water may suddenly increase and reach reservoir bodies very quickly. These observations may explain the increase of bacterial contamination during the month of September (which remained consistently high until April of the following year) in all sampling sites. The bacterial counts decreased drastically during the months of May, June, and July, which is winter (dry) season in South Africa. Similar findings were reported from studies by Shar et al. [42], Aertgeerts et al. [43], and Zia et al. [44]. The increase in microbial loads during the wet season resulted in an increase in the probability of infection/illness posed by both E. coli and Enterococcus (Fig. 3). Taking into consideration the increase in human beach activities during this period, especially contact with recreational water, the calculated $E$. coli risk of 
0.15 ( 1 in 10 people getting infected) and Enterococcus risk of 0.4 ( 4 in 10 people getting ill) may actually be representing quite a large number of cases, especially if compared to the WHO [26] "acceptable risk" of $1 \times 10^{-4}$ for recreational water. A 1 in 10 ratio translates to a 10 in 100 cases or 100 in 1000 cases, which can be way too high considering that the calculated risk does not take into consideration bather-to-bather transmission via the water (gastrointestinal illness) and air (respiratory pathogens). Fewtrell and Kay [45] reported that besides the risk of infection caused by organisms naturally present in the water, other causes of infection and illness are a result of direct transmission among bathers especially in overcrowded beaches.

In addition to microbial pathogens, urine and feces add up to beach contamination while in use by recreationists. Ideally, heterotrophic bacteria respond to organic pollution by decomposing and mineralizing organic matter to inorganic nutrients. Vignesh et al. [46] maintain that as human use of coastal landform increases, the incidence of contaminated water and consumption of shellfish result in subsequent aquaticborne diseases. On the other hand, it should be noted that the faecal origin of the bacteria is not indicated by the existence of faecal coliforms in the water source [47]. For this reason, enterococci are commonly used as a supplementary faecal indicator due to their ubiquity in human faeces and tenacity in the environment [48, 49]. Enterococci may be better indicators of human faecal pollution in water than $E$. coli $[50,51]$, and better indicators of threats to swimmers in contact with beach water in comparison to $E$. coli because these microorganisms survive longer in saline environments [47, 52-53].

In studies that focused on domestic and recreational water use in rural communities in developing countries [54-55], there have been issues of poor monitoring of water quality for more than a decade. This study has highlighted some of the challenges faced by public health, the environment, and water resources in South Africa and other developing countries. The possible implications could be outbreaks of water-borne diseases and the spread of pathogen resistance to antimicrobial agents in the presumptive. There is a high risk of infectious diseases such as diarrhea and cholera by bathers, tourists, recreationists, or visitors to recreational facilities. This study also covers identifying the influence of pathogen loading events and the importance of the epidemiology of incidence of infection on surface water quality.

\section{Conclusions}

From our findings we can conclude that the microbial quality of Nahoon Beach and its canal waters in the Eastern Cape Province is poor and did not conform to the set physicochemical and microbiological guidelines. There is therefore a need for frequent monitoring of beach water quality with standard methods. This study and other similar research works could assist the environmental protection agencies and health advisory with useful information if there are elevated levels of bacteria until pollution is controlled. More studies should also be encouraged with respect to recreational waters because of the few contributions that have been made, most especially the emergence of multidrugresistant microorganisms in beach water as well as beach sands.

\section{Acknowledgements}

This work was funded by SAMRC (South African Medical Research Council). The authors are indebted to anonymous reviewers for their valuable comments.

\section{Conflict of Interest}

The authors declare that there is no conflict of interest regarding the publication of this paper.

\section{References}

1. ABDELZAHER A.M., SOLO-GABRIELE H.M., PHILLIPS M.C., ELMIR S.M., FLEMING L.E. An Alternative Approach to Water Regulations for Public Health Protection at Bathing Beaches. Journal of Environmental and Public Health. 1, 2013.

2. ABDELZAHER A.M., WRIGHT M.E., ORTEGA C., SOLO-GABRIELE H.M., MILLER G., ELMIR S., FLEMING L.E. Presence of Pathogens and Indicator Microbes at a Non-Point Source Subtropical Recreational Marine Beach. Applied and Environmental Microbiology. 76 (724-732), 2010.

3. AHMAD A., DADA A.C., USUP G., HENG L.Y. Validation of the Enterococci indicator for bacteriological quality monitoring of beaches in Malaysia using a multivariate approach. SpringerPlus. 2 (1-18), 2013.

4. CABRAL J.P. Water Microbiology: Bacterial Pathogens and Water. International Journal of Environmental Research and Public Health. 7 (3657-3703), 2010.

5. BYAPPANAHALLI M.N., NEVERS M.B., KORAJKICA., STALEY Z.R., HARWOOD V.J. Enterococci in the Environment. Microbiology and Molecular Biology Reviews. 76 (685-706), 2012.

6. ASHBOLT N.J. Microbial Contamination of Drinking Water and Human Health from Community Water Systems. Current Environmental Health Reports. 2 (95106), 2015.

7. DOREVITCH S., PRATAP P., WROBLEWSKI M., HRYHORCZUK D.O., LI H., LIU L.C. Health Risks of Limited-Contact Water Recreation. Environmental Health Perspectives. 120 (192-197), 2012.

8. DEFLORIO-BARKER S., WADE T.J., JONES R.M., FRIEDMAN L.S., WING C., DOREVITCH S. Estimated Costs of Sporadic Gastrointestinal Illness Associated with Surface Water Recreation: A Combined Analysis of Data from NEEAR and CHEERS Studies. Environmental Health Perspectives. 125 (215-222), 2017. 
9. IGBINOSA E.O., OBI C.L., OKOH A.I. Seasonal abundance and distribution of Vibrio species in the treated effluent of wastewater treatment facilities in suburban and urban communities of Eastern Cape Province, South Africa. The Journal of Microbiology. 49 (224-232), 2011.

10. COSA S., OKOH A.I. Prevalence of Culturable Marine Actinomycetes Genera in Near-Shore Sediments of Algoa Bay in the Eastern Cape Province of South Africa. Journal of Pure and Applied Microbiology. 6 (1111-1118), 2012.

11. VALLI S., SUVATHI S.S., AYSHA O.S., NIRMALA P., VINOTH K.P., REENA A. Antimicrobial potential of Actinomycetes species isolated from marine environment. Asian Pacific Journal of Tropical Biomedicine. 2 (469473), 2012.

12. DUDDU M.K., GUNTUKU G. Isolation and partial characterization of Actinomycetes from mangrove sediment sample. Journal of Global Biosciences. 4 (29212929), 2015.

13. SCHLEYER M.H. Assessment of Marine Biological Diversity and Habitats. Western Indian Ocean. 33, 2015

14. BUSINESSTECH. Here are South Africas blue flag beaches for 2016/https://businesstech.co.za/news/ general/139443/. 2017.

15. UNITED STATES ENVIRONMENTAL PROTECTION AGENCY. Bacteriological ambient water quality criteria for marine and fresh recreational waters. EPA 440/5-84002. U.S. Environmental Protection Agency, Office of Research and Development, Cincinnati, OH. 2012.

16. SHASH S.M., KAMEL M.M., AL-WASIFY R.S., SAMHAN F.A. Rapid detection and enumeration of coliforms and Escherichia coli in River Nile using membrane filtration technique. Environmental Biotechnology. 6 (6-10), 2010.

17. ADEFISOYE M.A., OKOH A.I. Ecological and Public Health Implications of the Discharge of MultidrugResistant Bacteria and Physicochemical Contaminants from Treated Wastewater Effluents in the Eastern Cape, South Africa. Water. 9 (562), 2017.

18. JACKSON C.R., FEDORKA-CRAY P.J., BARRETT J.B. Use of a genus-and species-specific multiplex PCR for identification of enterococci. Journal of clinical microbiology. 42 (3558-3565), 2004.

19. STEYN M, JAGALS P., GENTHE B. Assessment of microbial infection risks posed by ingestion of water during domestic water use and full-contact recreation in a mid-southern African region. Water Science and Technology. 50 (301-308), 2004.

20. HAAS C.N., ROSE J.B., GERBA C.P. Quantitative microbial risk assessment. New York: John Wiley \& Sons. 1999.

21. STONE D.L., HARDING A.K., HOPE B.K., SLAUGHTER-MASON S. Exposure Assessment and Risk of Gastrointestinal Illness Among Surfers. Journal of Toxicology and Environmental Health, Part A. 71 (16031615), 2008.

22. FEWTRELL L., BARTRAM J. Water Quality: Guidelines, Standards and Health. Risk Assessment and Management for Water-related Infectious Disease. 2001.

23. SUNGER N., HAAS C.N. Quantitative microbial risk assessment for recreational exposure to water bodies in Philadelphia. Water Environment Research. 87 (211-222), 2015.

24. ABIA A.L.K., UBOMBA-JASWA E., GENTHE B., MOMBA M.N.B. Quantitative microbial risk assessment (QMRA) shows increased public health risk associated with exposure to river water under conditions of riverbed sediment resuspension. Science of the Total Environment. 566 (1143-1151), 2016.

25. STRACHAN N., DOYLE M., KASUGA F., ROTARIU O., OGDEN I. Dose response modelling of Escherichia coli $\mathrm{O} 157$ incorporating data from foodborne and environmental outbreaks. Int. J. Food Microbiology. 103 (35-47), 2005.

26. BENKE K.K., HAMILTON A.J. Quantitative microbial risk assessment: uncertainty and measures of central tendency for skewed distributions. Stochastic Environmental Research and Risk Assessment. 22 (533539), 2008.

27. KISTEMANN T., SCHMIDT A., FLEMMING H.C. Post-industrial river water quality-Fit for bathing again? International Journal of Hygiene and Environmental Health. 219 (629-642), 2016.

28. WORLD HEALTH ORGANIZATION. Guidelines for safe recreational water environments: Coastal and fresh waters (Vol. 1). World Health Organization. 2003.

29. DEPARTMENT OF WATER AFFAIRS AND FORESTRY. South African water quality guidelines (Vol. $2)$, recreational water use ( $2^{\text {nd }} E d$.). Pretoria: Department of Water Affairs and Forestry. 1996.

30. MAYP'I $\triangle O Y$ A. Health risks associated with microbial contamination of seashore sand. 2015.

31. FARRANT S., HEWSON K. Contaminant characterisation of storm water discharge into Okeover Stream and treatment recommendations. Natural Resources Engineering Research Project. 2006 (7-63), 2006.

32. LIU G., BAKKER G.L., LI S., VREEBURG J.H.G., VERBERK J.Q.J.C., MEDEMA G.J., LIU W.T., VAN DIJK J.C. Pyrosequencing reveals bacterial communities in unchlorinated drinking water distribution system: an integral study of bulk water, suspended solids, loose deposits, and pipe wall biofilm. Environmental science and technology. 48 (5467-5476), 2014.

33. BAKER A., CUMBERLAND S.A., BRADLEY C., BUCKLEY C. AND BRIDGEMAN J. To what extent can portable fluorescence spectroscopy be used in the realtime assessment of microbial water quality? Science of the Total Environment. 532 (14-19), 2015.

34. BRADY A.M.G., BUSHON R.N., PLONA M.B. Predicting recreational water quality using turbidity in the Cuyahoga River, Cuyahoga Valley National Park, Ohio, 20047: U.S. Geological Survey Scientific Investigations Report. 16 (2009-5192), 2009.

35. DISSANAYAKE P., CLEMETT A., JAYAKODY P., AMERASINGHE P. Report on water quality survey and pollution in Kurunegala, Sri Lanka. WASPA Asia Project Report, 8. 2007.

36. TRICK J.K., STUART M., REEDER S. Contaminated groundwater sampling and quality control of water analyses. Environmental geochemistry: site characterization, data analysis and case histories. Elsevier, Amsterdam, 29, 2008

37. EL-MAGD I.A., EL-ZEINY A. Quantitative hyperspectral analysis for characterization of the coastal water from Damietta to Port Said, Egypt. The Egyptian Journal of Remote Sensing and Space Science. 17 (61-76), 2014.

38. KEMKER C. Turbidity, Total Suspended Solids and Water Clarity: Fundamentals of Environmental Measurements. Fondriest Environmental, Inc, 13. 2014.

39. POMA H.R., CACCIABUEA D.G., GARCÉA B., GONZOA E.E., RAJAL V.B. Towards a rational strategy 
for monitoring of microbiological quality of ambient waters. Sci Total Environ. 433 (98-109), 2012.

40. MCNINCH R.M., SINGH S., ROSE J.B. Recreation in natural water resources. Water and Health-Volume II, 213. 2009.

41. GÖRANSSON G., LARSON M., BENDZ D., ÅKESSON M. Mass transport of contaminated soil released into surface water by landslides (Gota River, SW Sweden). Hydrology and Earth System Sciences. 16 (1879), 2012.

42. SHAR A.H., KAZI Y.F., KANHAR N.A., SOOMRO I.H., ZIA S.M., GHUMRO P.B. Drinking water quality in Rohri city, Sindh, Pakistan. African Journal of Biotechnology. 9 (7102-7107), 2010.

43. AERTGEERTS R., SINISI L., WORLD HEALTH ORGANIZATION, UNITED NATIONS, ECONOMIC COMMISSION FOR EUROPE. Guidance on water supply and sanitation in extreme weather events. 2011.

44. ZIA H., HARRIS N.R., MERRETT G.V., RIVERS M., COLES N. The impact of agricultural activities on water quality: A case for collaborative catchment-scale management using integrated wireless sensor networks. Computers and electronics in agriculture. 96 (126-138), 2013.

45. FEWTRELL L., KAY D. Recreational Water and Infection: A Review of Recent Findings. Curr Environ Health Rep. 2 (85-94), 2015.

46. VIGNESH S., DAHMS H.U., EMMANUEL K.V., GOKUL M.S., MUTHUKUMAR K., KIM B.R., JAMES R.A. Physicochemical parameters aid microbial community? A case study from marine recreational beaches, Southern India. Environmental monitoring and assessment. 186 (1875-1887), 2014.

47. DADA A.C., AHMAD A., USUP G., HENG L.Y. Speciation and antimicrobial resistance of Enterococci isolated from recreational beaches in Malaysia. Environmental monitoring and assessment. 185 (15831599), 2013.
48. BOEHM A. B., SASSOUBRE L. M. Enterococci as indicators of environmental faecal contamination. 2014.

49. SOLLER J.A., EFTIM S., WADE T.J., ICHIDA A.M., CLANCY J.L., JOHNSON T.B., SCHWAB K., RAMIREZ-TORO G., NAPPIER S., RAVENSCROFT J.E. Use of quantitative microbial risk assessment to improve interpretation of a recreational water epidemiological study. Microbial Risk Analysis. 1 (2-11), 2016.

50. PAYMENT P., LOCAS A. Pathogens in water: value and limits of correlation with microbial indicators. Ground Water. 49 (4-11), 2011.

51. PACHEPSKY Y., SHELTON D., DORNER S., WHELAN G. Can E. coli or thermotolerant coliform concentrations predict pathogen presence or prevalence in irrigation waters? Critical reviews in microbiology. 42 (384-393), 2016.

52. TIEFENTHALER L.L., STEIN E.D., LYON G.S. Faecal indicator bacteria (FIB) levels during dry weather from Southern California reference streams. Environmental monitoring and assessment. 155 (477-492), 2009.

53. BADGLEY B.D., NAYAK B.S., HARWOOD V.J. The importance of sediment and submerged aquatic vegetation as potential habitats for persistent strains of enterococci in a subtropical watershed. Water research. 44 (5857-5866), 2010.

54. MCGARVEY S.T., BUSZIN J., REED H., SMITH D.C., RAHMAN Z., ANDRZEJEWSKI C., AWUSABOASARE K., WHITE M.J. Community and household determinants of water quality in coastal Ghana. Journal of water and health. 6 (339-349), 2008.

55. GALADIMA A., GARBA Z.N., LEKE L., ALMUSTAPHA M.N., ADAM I.K. Domestic water pollution among local communities in Nigeria-causes and consequences. European Journal of Scientific Research. 52 (592-603), 2011. 
